\title{
Article \\ Spread of Linezolid-Resistant Enterococcus spp. in Human Clinical Isolates in the Czech Republic
}

\author{
Lucia Mališová ${ }^{1,2}$, Vladislav Jakubů ${ }^{1,2,3}$, Katarína Pomorská $^{1}$, Martin Musílek ${ }^{4} \mathbb{D}$ and \\ Helena Žemličková 1,2,3,*(i)
}

1 National Reference Laboratory for Antibiotics, Centre for Epidemiology and Microbiology, National Institute of Public Health, 10000 Prague, Czech Republic; lucia.malisova@szu.cz (L.M.); vladislav.jakubu@szu.cz (V.J.); katarina.pomorska@szu.cz (K.P.)

2 Department of Microbiology, 3rd Faculty of Medicine Charles University, University Hospital Kralovske Vinohrady and National Institute of Public Health, 10000 Prague, Czech Republic

3 Department of Clinical Microbiology, Faculty of Medicine and University Hospital, Charles University, 53002 Hradec Kralove, Czech Republic

4 National Reference Laboratory for Meningococcal Infections, Centre for Epidemiology and Microbiology, National Institute of Public Health, 10000 Prague, Czech Republic; martin.musilek@szu.cz

* Correspondence: hzemlickova@szu.cz

Citation: Mališová, L.; Jakubů, V.;

Pomorská, K.; Musílek, M.;

Žemličková, H. Spread of

Linezolid-Resistant Enterococcus spp. in Human Clinical Isolates in the Czech Republic. Antibiotics 2021, 10, 219. https://doi.org/10.3390/ antibiotics 10020219

Academic Editor: Jeffrey Lipman

Received: 27 January 2021

Accepted: 20 February 2021

Published: 22 February 2021

Publisher's Note: MDPI stays neutral with regard to jurisdictional claims in published maps and institutional affiliations.

\begin{abstract}
The aim of this study was to map and investigate linezolid resistance mechanisms in linezolid-resistant enterococci in the Czech Republic from 2009 to 2019. Altogether, 1442 isolates of Enterococcus faecium and Enterococcus faecalis were examined in the National Reference Laboratory for Antibiotics. Among them, $8 \%$ of isolates $(n=115)$ were resistant to linezolid $($ E. faecium $/ n=106, E$. faecalis $/ n=9$ ). Only three strains of E. faecium were resistant to tigecycline, $72.6 \%$ of isolates were resistant to vancomycin. One isolate of E. faecium harbored the $c f r$ gene. The majority $(87 \%, n=11)$ of E. faecium strains were resistant to linezolid because of the mutation G2576T in the domain V of the $23 S$ rRNA. This mutation was detected also in two strains of E. faecalis. The presence of the optrA gene was the dominant mechanism of linezolid resistance in E. faecalis isolates. None of enterococci contained $c f r B$, poxt $A$ genes, or any amino acid mutation in genes encoding ribosomal proteins. No mechanism of resistance was identified in 4 out of $106 \mathrm{E}$. faecium linezolid resistant isolates in this study. Seventeen sequence types (STs) including four novel STs were identified in this work. Clonal complex CC17 was found in all E. faecium isolates.
\end{abstract}

Keywords: Enterococcus faecium; Enterococcus faecalis; linezolid resistance; $23 \mathrm{~S}$ rRNA; optrA

\section{Introduction}

Enterococci are Gram-positive bacteria, commensals of the gastrointestinal tract and opportunistic pathogens able to cause community-acquired and nosocomial infections. Two species, Enterococcus faecium and Enterococcus faecalis, are considered to be one of the most important nosocomial pathogens worldwide [1]. They cause life-threatening infections especially in elderly, polymorbid and immunocompromised patients [2]. Increasing resistance of enterococci to penicillin, aminoglycosides, glycopeptides or to the last resort antibiotics (daptomycin, tigecycline, linezolid) prevents these drugs from being effective in the treatment of infections caused by these bacteria [3].

Linezolid is a bacteriostatic antibiotic efficient only against Gram-positive bacteria including methicillin-resistant Staphylococcus aureus (MRSA) and vancomycin resistant enterococci [4]. It inhibits the accuracy of the protein translation by binding to the peptidyl transferase centrum (PTC) in the V domain of the 23S rRNA inside the 50S ribosomal subunit [5]. Since the introduction of linezolid into clinical use in 2000 (USA) [4], seven mechanisms have been described as related to the linezolid resistance in enterococci: $\mathrm{Mu}-$ tations in the 23S rRNA and genes encoding ribosomal proteins L3, L4, and L22, the 
acquisition of plasmid carrying genes cfr (chloramphenicol-florfenicol) [6], optrA (ABC transporter that confers resistance to oxazolidinones and phenicols) [7] and poxt $A$ (ABC transporter; resistance to oxazolidinones, phenicols and tetracyclines) [8]. The most common mechanism of linezolid resistance in enterococci is the conversion of $\mathrm{G}$ to $\mathrm{T}$ at position 2576 in the $23 \mathrm{~S}$ rRNA [9]. Ribosomal proteins L3, L4, L22 play an important role in the stabilization and conformation of the ribosome (PTC). Therefore, mutations in genes $(r p l C$, $r p l D$, and $r p l V$ ) encoding these proteins lead to the amino acid changes followed by disruption of translation. This type of mechanism is predominantly linked with linezolid resistance in Staphylococcus epidermidis [10].

The gene $c f r$ encodes a methyltransferase that catalyzes the posttranscriptional methylation of nucleotide A2503 in the $23 \mathrm{~S}$ rRNA [10]. It has been described for the first time in S. aureus in 2005 [11], and it can be transferred across different bacterial species and genera [12]. New mechanisms, optrA (China, 2015) [7] and poxtA [8], belonging to the ABC-F family of ATP-binding cassette (efflux pump genes) were revealed recently. Comparative analysis at the protein levels in the genome of linezolid resistant $S$. aureus revealed $32 \%$ protein homology between them [8]. Gene optrA has been more often detected in livestock than in humans and its occurrence is more associated with E. faecalis than $E$. faecium strains [1]. PoxtA was originally identified in Italian isolate MRSA in 2018 [8] and its prevalence amongst the enterococcal population is still under investigation.

The purpose of this study was to investigate the spread of linezolid resistant enterococci acquired from human clinical specimens in the Czech Republic over the period of 10 years, and to analyze molecular mechanisms of their resistance to linezolid.

\section{Results}

\subsection{Antibiotic Susceptibility of Linezolid Resistant Isolates of E. faecium and E. faecalis}

Altogether, 1442 enterococcal isolates (791/58.5\%/E. faecium, 651/45\%/E. faecalis) were examined in the National Reference Laboratory for Antibiotics from 2009 to 2019. Of them, 115 strains (8\%) were resistant to linezolid: 106 isolates (13.4\%) of E. faecium and 9 strains $(1.4 \%)$ of $E$. faecalis. The number of linezolid resistant enterococci increased from 2009 to 2019 (E. faecium; from 2009/0 to 2019/32, E. faecalis; from 2009/0 to 2019/4, Supplementary Figure S1). Resistance to vancomycin was confirmed in $72.6 \%(n=77)$ of E. faecium strains. Resistance to teicoplanin was detected in $70 \%(n=74)$ of isolates, $2.8 \%(n=3)$ of strains were resistant to tigecycline. The majority of linezolid resistant $E$. faecium isolates were resistant also to gentamicin $(76.5 \%, n=89)$ and streptomycin $(69 \%, n$ $=79$ ). None of linezolid resistant $E$. faecalis strains was resistant to ampicillin, teicoplanin, vancomycin and tigecycline. All of them were resistant to gentamicin, 4 isolates also to streptomycin (Table 1, Supplementary Table S1).

\subsection{Mechanisms of Linezolid and Vancomycin Resistance}

Altogether, $93.4 \%(n=99)$ of E. faecium isolates harbored the point mutation G2576T in the $\mathrm{V}$ domain of the $23 \mathrm{~S} \mathrm{rRNA}$, two strains of E. faecium were positive for the presence of optrA gene, one isolate was cfr positive. There was not revealed the mechanism of linezolid resistance in four isolates. In 7 out of 9 E. faecalis isolates, the presence of optrA gene was confirmed, the mutation G2576T was revealed in two samples. Resistance to vancomycin was detected only in E. faecium strains. The presence of $\operatorname{van} A(92 \%, n=71)$, and $\operatorname{vanB}(n=2)$ genes was confirmed. Four isolates were resistant to vancomycin due to the combination of $\operatorname{van} A$, vanB $(n=4)$ genes (Table 1, Supplementary Table S1). 


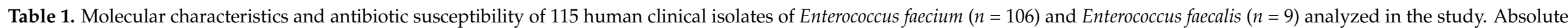
numbers are expressed in \% (number of isolates) and depict the number of resistant population in the group.

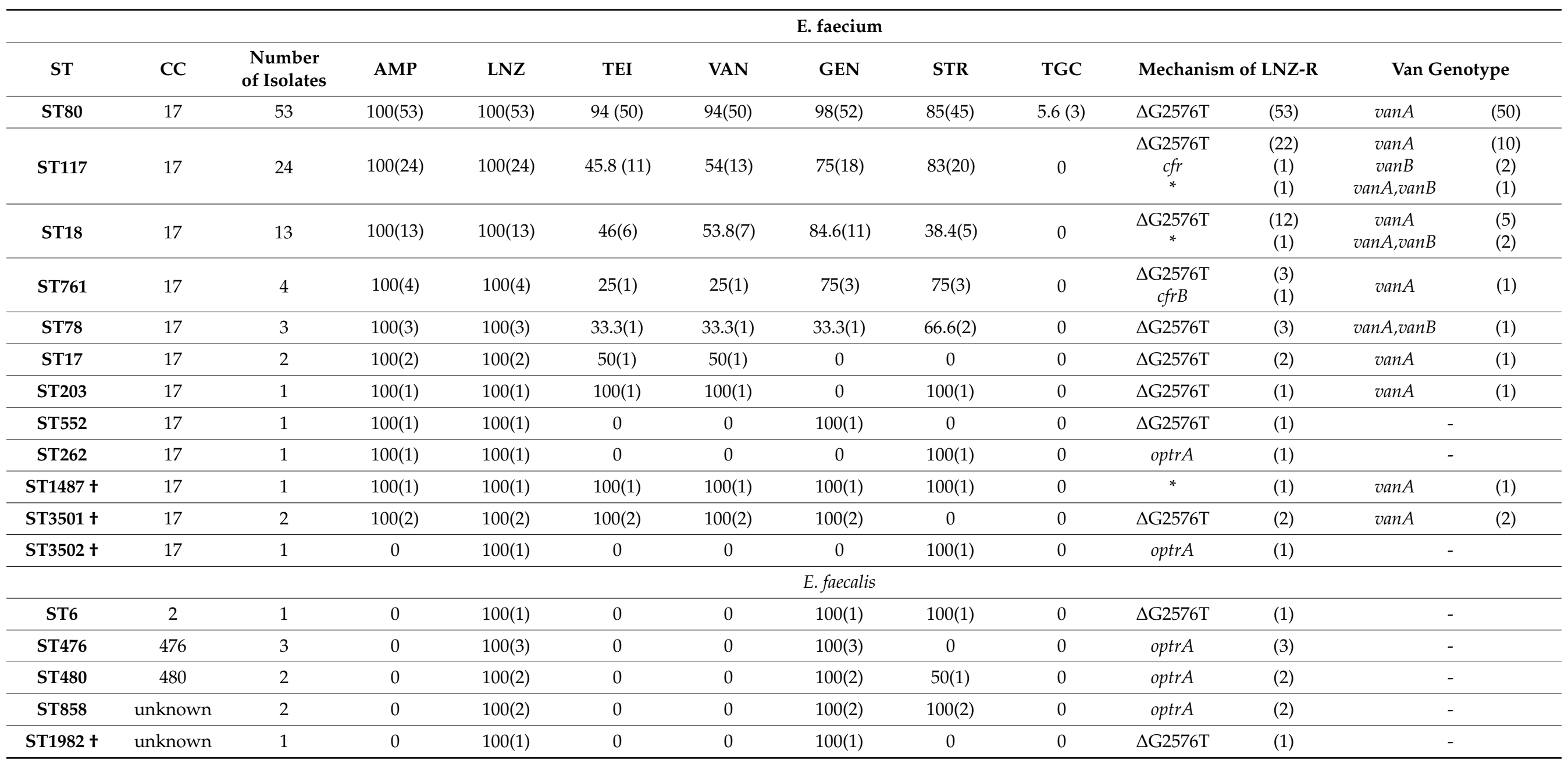

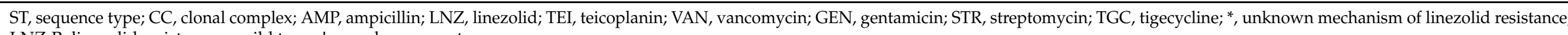
LNZ-R, linezolid resistance; -, wild type;,+ novel sequence types. 


\subsection{Molecular Typing-MLST Analysis}

Altogether, 12 different sequence types (STs) were found in linezolid resistant $E$. faecium strains. The most frequent STs detected were ST80 $(n=53)$ and ST117 $(n=24)$, followed by ST18 $(n=13)$, ST761 $(n=4)$, ST78 $(n=3)$. Other STs were represented by a single or two isolates: ST17 $(n=2)$, ST203 $(n=1)$, ST552 $(n=1), \operatorname{ST} 262(n=1)$. Due to the new type of gyd allele, three novel STs were identified in the study: ST1487 $(n=1)$, ST3501 $(n=2)$, ST3502 $(n=1)$ (Supplementary Figure S2). Almost all strains of E. faecium $(97 \%, n=$ 103) belonged to the same clonal complex, CC17 (Table 1, Supplementary Table S1). MLST typing revealed a high genetic variability within the group of $E$. faecalis strains. Altogether, there were confirmed 5 STs: ST6/CC2 $(n=1) /$, ST476/CC476 $(n=3)$, ST480 $(n=2)$, ST858 $(n=2)$ and a new ST1982 $(n=1)$ (Supplementary Figure S3).

\section{Discussion}

This is the first study mapping linezolid resistant enterococci acquired from human clinical specimen in the Czech Republic. Enterococci have become one of the most prevalent (nosocomial) pathogens over the past decades and linezolid provides one of the therapeutic options for infections caused by this bacteria.

Among 1442 enterococci sent to the National Reference Laboratory for Antibiotics from 2009 to 2019, 8\% of them were resistant to linezolid. Congruently with other European countries, the pattern of occurrence of linezolid resistant enterococci increased from year to year $(0 / 2009-36 / 2019)[9,13]$. Except for 3 strains $(2.6 \%)$ almost all analyzed enterococci were susceptible to tigecycline, an alternative option in the treatment of enterococcal infections. In accordance with this observation, the majority of the European countries reports generally low prevalence $(<1 \%)$ of isolates resistant to last-resort antibiotics (daptomycin, tigecycline, linezolid) [14]. A lower rate of linezolid resistance in enterococci could be explained by lower selection pressure of this antibiotic or by its mechanism. The substitution of $\mathrm{G}$ to $\mathrm{T}$ in the position 2576 in the 23S rRNA develops as a spontaneous mutation. It was reported that the frequency of the spontaneous resistance to linezolid (in enterococci) is lower than to other antibiotics [15]. This statement has been confirmed also in this work, $87.8 \%(101 / 115)$ of linezolid resistant enterococci contained the mutation G2576T in the $23 \mathrm{~S}$ rRNA [16]. Mutations in $r p l C, r p l D$, and $r p l V$ genes were not observed in this work. Mutations in genes encoding ribosomal proteins (L3, L4, L22) were more commonly seen in coagulase-negative staphylococci (preferentially S. epidermidis) than in enterococci [17].

The rate of E. faecium isolates harboring gene optrA was negligible. Altogether, only two isolates (ST262, ST3502) were positive for the optrA gene. This result is not surprising, the occurrence of optrA has been associated more with E. faecalis than E. faecium species $[18,19]$. The presence of the gene optr $A$ was the dominant mechanism responsible for the linezolid resistance in E. faecalis strains. It is worth noting, that optrA positive linezolid resistant enterococci can confer MICs of linezolid different than other linezolid resistant enterococci [20]. All enterococci positive for the gene optr $A$ were associated with MICs for linezolid at the level up to $8 \mathrm{mg} / \mathrm{L}$. The majority of enterococci harboring the G2576T mutation had MICs $\geq 16 \mathrm{mg} / \mathrm{L}$.

None from enterococci analyzed in this work harbored the gene poxt $A$. This, a novel antibiotic resistance determinant, was until now identified only in one clinical isolate of E. faecium in Greece in 2018 [21]. Despite the sporadic occurrence of the poxt $A$, the scattered distribution of this gene among the different Gram-positive species (e.g., S. aureus, Enterococcus spp., etc.) deserves an attention [22].

At present, eight different types of van genes conferring vancomycin resistance mechanisms are known. The van $A$ and $\operatorname{van} B$ resistance genotypes are the most frequently detected variants in clinical isolates of E. faecium and E. faecalis worldwide [14]. Vancomycin resistance caused by the presence of gene van $A$ was a dominant mechanism in the group of vancomycin resistant enterococci in the Czech Republic. Due to the location of the gene van $A$ on plasmid, this fragment of DNA can spread easier than vanB, which is usually a part of the bacterial chromosome [23]. Based on the global spread of enterococci resistant 
to the different kinds of antibiotics (including the last resort antibiotics), a molecular epidemiology studies are performed to obtain insights into the dissemination of these strains. MLST is a molecular typing method with suitable discriminatory power [23] very often used for this purpose. One pandemic clone of CC17 was detected in linezolid resistant E. faecium isolates in the Czech Republic from 2009 to 2019. The enterococcal lineage of CC17 is responsible for the spread of linezolid and vancomycin resistance in hospitals all around the world [1]. Moreover, strains belonging to this clonal complex show persistence in the environment and high colonization capability [24]. In this study, linezolid resistant enterococci belonged to 12 different STs, but a majority (50\%) of isolates typed as ST80 was observed [25]. ST80 was described for the first time in blood of Israeli patient in 1997 (unpublished data, the source: pubmlst.org). Since then it has been detected all over the world. ST18, ST78, and ST117 were associated with enterococci also in other European countries [24,25]. ST17, ST203, and ST552 were observed earlier by Egan et al. in Ireland [26]. In concordance with others, a high risk STs associated with linezolid resistance in enterococci involved also ST262 [27], ST761 [28] and newly identified STs 3501, 3502, 1487 and 1982. A classical hospital-associated CC2 (ST6) of E. faecalis has been already detected in Spain and Poland [29,30]. STs 585 and 476 have been observed in overall diverse population of optrA-positive E. faecalis strains in Portugal [31].

Inability to infer the linezolid resistance mechanism in three E. faecium isolates suggests the possibility of presence of additional mechanisms of resistance. It is supported by results of studies on enterococci with a silent mechanism of resistance, but still exhibiting linezolid resistance [32]. Enterococci are adaptable bacteria characterized by a high plasticity of genome (a high rate of DNA recombination). Therefore, novel mechanisms of resistance to different kinds of antibiotics have emerged relatively rapidly. Efflux pumps, cell wall thickness and biofilm formation are still discussed as putative alternative pathways of linezolid resistance [33,34].

In conclusion, this study provided the first insight into the population structure of linezolid resistant enterococci in the Czech Republic within the period of 10 years. It showed that the rate of linezolid resistant enterococci was comparable with other European countries and it increased in both groups of examined enterococci. The main mechanism of linezolid resistance among clinical E. faecium isolates was the G2576T mutation in the domain $\mathrm{V}$ of the $23 \mathrm{~S}$ rRNA. The presence of gene optrA was the major cause of linezolid resistance in E. faecalis strains. A high risk clone CC17 was the only CC detected in linezolid resistant E. faecium strains in the Czech Republic within last decade.

Still increasing prevalence of enterococci resistant to the last resort antibiotics as well as their ability to acquire novel DNA fragments encoding (new) resistance determinants predestine these bacteria to even more successful spreading. Therefore, enterococci resistant to linezolid represents a public health concern and monitoring the spread of these bacteria is necessary.

\section{Materials and Methods}

\subsection{Bacterial Isolates}

Screening of E. faecium/E. faecalis strains is performed as a part of European Antimicrobial Resistance Surveillance Network (EARS-Net) and the study of Monitoring of Antibiotic Resistance in the National Reference Laboratory for Antibiotics (National Institute of Public Health, Prague, Czech Republic). Enterococci presented in this study were acquired from 40 laboratories in the period from 2009 to 2019. The majority of strains, $61 \%(n=875)$, was of invasive origin (blood; $n=873$, cerebrospinal fluid; $n=2)$. The rest of isolates was acquired form non-invasive clinical specimens, and involved surgical wound, urine, pus, sputum, catheter, aspirate, bile, and swab (mouth, throat, nose, and vagina). Altogether, $5 \%(n=70)$ of enterococci were isolated from rectal swab and stool sample. One isolate was of unknown origin. Characteristics of linezolid resistant strains are given in the Supplementary Table S1. Enterococci resistant to linezolid are further examined to reveal the mechanism of linezolid resistance and their epidemiological relationship 
(MLST). Resistance to linezolid ( $\geq 4 \mathrm{mg} / \mathrm{L}$ ) was defined according to the European Committee on Antimicrobial Susceptibility Testing (EUCAST) breakpoint (www.eucast.org). All strains were routinely cultivated on Columbia blood agar (Oxoid, Brno, Czech Republic) aerobically at $36 \pm 1{ }^{\circ} \mathrm{C}$. Identification of strains was performed by Matrix-Assisted Laser Desorption Ionization-Time of Flight Mass Spectrometry (MALDI-TOF MS; Microflex Bruker, Bremen, Germany) according to the manufacturer's protocol (www.bruker.com).

\subsection{Susceptibility Testing}

Minimal inhibitory concentrations (MICs) of ampicillin, linezolid, teicoplanin, vancomycin, gentamicin, streptomycin, and tigecycline were determined by broth microdilution method according to ISO 20776-1. Interpretation of susceptibility testing results was performed as recommended by EUCAST, version according to a corresponding year (last used version 9.0). Strains ATCC 29212 and ATCC 51299 were used as quality control in this study (both strains recommended by EUCAST; www.eucast.org).

\subsection{Detection of Determinants of Linezolid Resistance}

Mechanism of resistance to linezolid was determined by PCR ( $c f r, c f r B$, oprA, and poxtA) and Sanger sequencing (23S rRNA, $r p l C, r p l D$, and $r p l V$ ). DNA extraction was obtained from a fresh culture (24 hours) according to the manufacturer's protocol (GenElute TM Bacterial Genomic DNA Kits, Sigma Aldrich, St. Louis, MO, USA). The list of enterococcal specific primers and settings of PCR used in the study are given in the Supplementary Table S2. PCR products of $c f r$, oprA were resolved in $1.5 \%$ agarose (TopVision Agarose, Thermo Scientific, St. Louis, MO, USA) in electrophoresis $(5 \mathrm{~V} / \mathrm{cm})$ for 45 minutes. NCTC13923 was used as control strain for optrA detection in this study. The detection of $c f r$, poxt $A$, and $c f r B$ was performed according to procedures as described previously $[8,12,35]$. The amplified fragments of the $23 \mathrm{~S}$ rRNA, $r p l C, r p l D$, and $r p l V$ were sequenced by analyzer Applied Biosystems 3130xL. Point mutation/s associated with linezolid resistance were analyzed using software Bionumerics 7.6.2 (Applied Maths, Ghent, East Flanders, Belgium).

\subsection{Detection of Mechanism of Vancomycin Resistance}

Isolates resistant to linezolid and simultaneously resistant to vancomycin were further examined. The mechanism of resistance to vancomycin was screened by PCR using primers under conditions that are listed in the Supplementary Table S2. Electrophoresis was carried out as described above.

\subsection{MLST Typing of Linezolid Resistant Strains of E. faecium, E. faecalis}

Epidemiology of enterococci was investigated by the multilocus sequence typing (MLST) analysis as described earlier [36]. Seven primers targeting adk (adenylate kinase), $\operatorname{atp} A$ (ATP synthase, alpha subunit), $d d l$ (d-alanine:d-alanine ligase), gyd (glyceraldehyde3-phosphate dehydrogenase), gdh (glucose-6-phosphate dehydrogenase), purK (phosphoribosylaminoimidazol carboxylase ATPase subunit) and pstS (phosphate ATP-binding cassette transporter) alleles were used to amplify target region in E. faecium isolates. PstS (phosphate ATP binding cassette transporter), gki (putative glucokinase), aroE (shikimate 5-dehydrogenase), xpt (shikimate 5-dehydrogenase), gyd, gdh, and yiqL (acetyl-coenzyme A acetyltransferase) were used for MLST analysis of E. faecalis strains. Alleles gyd and gdh were amplified using the same primers for both species. The list of primers used for MLST analysis of enterococci is given in the Supplementary Table S3. All sequences were processed by analyser (Applied Biosystems 3130xL, Foster City, CA, USA). Allelic profiles, sequence types (STs) and clonal complexes (CC) of enterococci were determined using Bionumerics 7.6.2 (Applied Maths, Ghent, East Flanders, Belgium) and free available website pubmlst.org (https:/ / pubmlst.org/organisms).

Supplementary Materials: The following are available online at https:/ / www.mdpi.com/2079-638 2/10/2/219/s1, Figure S1: Detection of linezolid resistant enterococci acquired from human clinical specimen in the Czech Republic from 2009 to 2019, Figure S2: STs occurrence in the group of linezolid 
resistant E. faecium ( $n=106$ ) strains from 2009 to 2019, Figure S3: STs occurrence in the group of linezolid resistant E. faecalis $(n=9)$ isolates from 2009 to 2019, Table S1: Phenotypic and genotypic characteristics of the 115 linezolid-resistant enterococcal isolates examined in the NRL for ATB between 2009 and 2019, Table S2: Primers used in the study, Table S3: Primers used in the MLST analysis of E. faecium and E. faecalis isolates.

Author Contributions: L.M. methodology, data analysis, witting-original draft preparation; V.J. writing - review and editing; K.P. writing—review and editing; M.M. methodology; H.Ž. writingreview and editing, project administration. All authors have read and agreed to the published version of the manuscript.

Funding: This research was funded by Ministry of Health, Czech Republic-conceptual development of research organization the National Institute of Public Health-NIPH, 75010330.

Data Availability Statement: The data presented in this study are available on request from the corresponding author.

Acknowledgments: Authors appreciate technical assistance of Jiří Kašík and Markéta Čechová. We would like to thank all EARS-Net participating laboratories for sending strains to the National Reference Laboratory for Antibiotics.

Conflicts of Interest: The authors declare no conflict of interest.

\section{References}

1. Prieto, A.M.; van Schaik, W.; Rogers, M.R.C.; Coque, T.M.; Baquero, F.; Corander, J.; Willems, R.J.L. Global Emergence and Dissemination of Enterococci as Nosocomial Pathogens: Attack of the Clones? Front. Microbiol. 2016, $26,788$.

2. Arias, C.A.; Murray, B.E. The rise of the Enterococcus: Beyond vancomycin resistance. Nat. Rev. Microbiol. 2012, 10, 266-278. [CrossRef] [PubMed]

3. Murray, B.E. The life and times of the Enterococcus. Clin. Microbiol. Rev. 1990, 3, 46-65. [CrossRef]

4. Mendes, R.E.; Deshpande, L.M.; Jones, R.N. Linezolid update: Stable in vitro activity following more than a decade of clinical use and summary of associated resistance mechanisms. Drug Resist. Updates 2014, 17, 1-12. [CrossRef]

5. Bourgeois-Nicolaos, N.; Massias, L.; Couson, B.; Butel, M.J.; Andremont, A.; Doucet-Populaire, F. Dose Dependence of Emergence of Resistance to Linezolid in Enterococcus faecalis In Vivo. J. Infect. Dis. 2007, 10, 1480-1488. [CrossRef] [PubMed]

6. Diaz, L.; Kiratisin, P.; Mendes, R.E.; Panesso, D.; Singh, K.V.; Arias, C.A. Transferable plasmid-mediated resistance to linezolid due to cfr in a human clinical isolate of Enterococcus faecalis. Antimicrob. Agents Chemother. 2012, 56, 3917-3922. [CrossRef]

7. Wang, Y.; Lv, Y.; Cai, J.; Schwarz, S.; Cui, L.; Hu, Z.; Zhang, R.; Li, J.; Zhao, Q.; He, T.; et al. A novel gene, optrA that confers transferable resistance to oxazolidinones and phenicols and its presence in Enterococcus faecalis and Enterococcus faecium of human and animal origin. J. Antimicrob. Chemother. 2015, 70, 2182-2190. [CrossRef]

8. Antonelli, A.; D'Andrea, M.M.; Brenciani, A.; Galeotti, C.L.; Morroni, G.; Pollini, S.; Varaldo, P.E.; Rossolini, G.M. Characterization of poxtA, a novel phenicol-oxazolidinone-tetracycline resistance gene from an MRSA of clinical origin. J. Antimicrob. Chemother. 2018, 73, 1763-1769. [CrossRef] [PubMed]

9. Klare, I.; Fleige, C.; Geringer, U.; Thurmer, A.; Bender, J.; Mutters, N.T.; Mischnik, A.; Werner, G. Increased frequency of linezolid resistance among clinical Enterococcus faecium isolates from German hospital patients. J. Glob. Antimicrob. Resist. 2015, 3, 128-131. [CrossRef] [PubMed]

10. Patel, S.N.; Memari, N.; Shahinas, D.; Toye, B.; Jamieson, F.B.; Farrell, D.J. Linezolid resistance in Enterococcus faecium isolated in Ontario, Canada. Diagn. Microbiol. Infect. Dis. 2013, 77, 350-353. [CrossRef] [PubMed]

11. Rossney, A.S.; Shore, A.C.; Morgan, P.M.; Fitzgibbon, M.M.; O'Connell, B.; Coleman, D.C. The emergence and importation of diverse genotypes of methicillin-resistant Staphylococcus aureus (MRSA) harboring the Panton-Valentine leukocidin gene (pvl) reveal that pvl is a poor marker for community-acquired MRSA strains in Ireland. J. Clin. Microbiol. 2007, 45, 2554-2563. [CrossRef]

12. Bender, J.K.; Fleige, C.; Klare, I.; Fiedler, S.; Mischnik, A.; Mutters, N.T.; Dingle, K.E.; Werner, G. Detection of a cfr(B) Variantin German Enterococcus faecium Clinical Isolates and the Impact on Linezolid Resistance in Enterococcuss pp. PLoS ONE 2016, 11, e0167042. [CrossRef] [PubMed]

13. Moure, Z.; Lara, N.; Marín, M.; Sola-Campoy, P.J.; Bautista, V.; Gómez-Bertomeu, F.; Gómez-Dominguez, C.; Pérez-Vázquez, M.; Aracil, B.; Campos, J.; et al. Spanish Linezolid-Resistant Enterococci Collaborating Group, Interregional spread in Spain of linezolid-resistant Enterococcus spp. isolates carrying the optrA and poxtA genes. Int. J. Antimicrob. Agents 2020, 55, 105977. [CrossRef] [PubMed]

14. Bender, J.K.; Cattoir, V.; Hegstad, K.; Sadowy, E.; Coque, T.M.; Westh, H.; Hammerum, A.M.; Schaffer, K.; Burns, K.; Murchan, S.; et al. Update on prevalence and mechanisms of resistance to linezolid, tigecycline and daptomycin in enterococci in Europe: Towards a common nomenclature. Drug Resist. Updates 2018, 40, 25-39. [CrossRef] 
15. Prystowsky, J.; Siddiqui, F.; Chosay, J.; Shinabarger, D.L.; Millichap, J.; Peterson, L.R.; Noskin, G.A. Resistance to linezolid: Characterization of mutations in rRNA and comparison of their occurrences in vancomycin-resistant enterococci. Antimicrob. Agents Chemother. 2001, 45, 2154-2156. [CrossRef] [PubMed]

16. Ntokou, E.; Stathopoulos, C.; Kristo, I.; Dimitroulia, E.; Labrou, M.; Vasdeki, A.; Makris, D.; Zakynthinos, E.; Tsakris, A. Intensive care unit dissemination of multiple clones of linezolid-resistant Enterococcus faecalis and Enterococcus faecium. J. Antimicrob. Chemother. 2012, 67, 1819-1823. [CrossRef]

17. Mendes, R.E.; Deshpande, L.M.; Costello, A.J.; Farrell, D.J. Molecular epidemiology of Staphylococcus epidermidis clinical isolates from U.S. hospitals. Antimicrob. Agents Chemother. 2012, 56, 4656-4661. [CrossRef]

18. Cavaco, L.M.; Bernal, J.F.; Zankari, E.; Le'on, M.; Hendriksen, R.S.; Perez-Gutierrez, E.; Aarestrup, F.M.; Donado-Godoy, P. Detection of linezolid resistance due to the optrA gene in Enterococcus faecalis from poultry meat from the American continent (Colombia). J. Antimicrob. Chemother. 2017, 72, 678-683. [PubMed]

19. Vorobieva, V.; Roer, L.; Justesen, U.S.; Hansen, F.; Frimodt-Moller, N.; Hasman, H.; Hammerum, A.M. Detection of the optrA gene in a clinical ST16 Enterococcus faecalis isolate in Denmark. J. Glob. Antimicrob. Resist. 2017, 10, 12-13. [CrossRef]

20. Ruiz-Ripa, L.; Feßler, A.T.; Hanke, D.; Eichhorn, I.; Azcona-Gutiérrez, J.M.; Pérez-Moreno, M.O.; Seral, C.; Aspiroz, C.; Alonso, C.A.; Torres, L.; et al. Mechanisms of Linezolid Resistance Among Enterococci of Clinical Origin in Spain-Detection of optrA- and cfr(D)-Carrying E. faecalis. Microorganisms 2020, 8, 1155. [CrossRef]

21. Papagiannitsis, C.C.; Tsilipounidaki, K.; Malli, E.; Petinaki, E. Detection in Greece of a clinical Enterococcus faecium isolate carrying the novel oxazolidinone resistance gene poxtA. J. Antimicrob. Chemother. 2019, 8, 2461-2462. [CrossRef] [PubMed]

22. Wu, Y.; Fan, R.; Wang, Y.; Lei, L.; Feßler, A.T.; Wang, Z.; Wu, C.; Schwarz, S.; Wang, Y. Analysis of combined resistance to oxazolidinones and phenicols among bacteria from dogs fed with raw meat/vegetables and the respective food items. Sci. Rep. 2019, 1, 15500. [CrossRef] [PubMed]

23. Werner, G.; Fleige, C.; Neumanna, B.; Bender, J.K.; Layer, F.; Klare, I. Evaluation of DiversiLab ${ }^{\circledR}$, MLST and PFGE typing for discriminating clinical Enterococcus faecium isolates. J. Microbiol. Methods 2015, 118, 81-84. [CrossRef] [PubMed]

24. Werner, G. Molecular Typing of Enterococci/VRE. Werner. J. Bacteriol. Parasitol. 2013, 10, 2155-9597. [CrossRef]

25. Kerschner, H.; Cabal, A.; Hartl, R.; Machherndl-Spandl, S.; Allerberger, F.; Ruppitsch, W.; Apfalter, P. Hospital outbreak caused by linezolid resistant Enterococcus faecium in Upper Austria. Antimicrob. Resist. Infect. Control 2019, 8, 150. [CrossRef]

26. Egan, S.A.; Shore, A.C.; O'Connell, B.; Brennan, G.I.; Coleman, D.C. Linezolid resistance in Enterococcus faecium and Enterococcus faecalis from hospitalized patients in Ireland: High prevalence of the MDR genes optrA and poxtA in isolates with diverse genetic backgrounds. J. Antimicrob. Chemother. 2020, 75, 1704-1711. [CrossRef]

27. Quiñones, D.; Aung, M.S.; Martins, J.P.S.; Urushibara, N.; Kobayashi, N. Genetic characteristics of VanA-type vancomycinresistant Enterococcus faecalis and Enterococcus faecium in Cuba. New Microbes New Infect. 2018, 21, 125-127. [CrossRef] [PubMed]

28. Wang, S.; Guo, Y.; Lv, J.; Qi, X.; Li, D.; Chen, Z.; Zhang, X.; Wang, L.; Yu, F. Characteristic of Enterococcus faecium clinical isolates with quinupristin/dalfopristin resistance in China. BMC Microbiol. 2016, 16, 246. [CrossRef]

29. Kawalec, M.; Pietras, Z.; Danilowicz, E.; Jakubczak, A.; Gniadkowski, M.; Hryniewicz, W.; Willems, R. Clonal structure of Enterococcus faecalis isolated from Polish hospitals: The characterization of epidemic clones. J. Clin. Microbiol. 2007, 1, 147-153. [CrossRef]

30. Ruiz-Garbajosa, P.; Bonten, M.J.; Robinson, D.A.; Top, J.; Nallapareddy, S.R.; Torres, C.; Coque, T.M.; Canton, R.; Baquero, F.; Murray, B.E.; et al. Multilocus sequence typing scheme for Enterococcus faecalis reveals hospital-adapted genetic complexes in a background of high rates of recombination. J. Clin. Microbiol. 2006, 44, 2220-2228. [CrossRef]

31. Freitas, A.R.; Tedim, A.P.; Novais, C.; Lanza, V.L.; Peixe, L. Comparative genomics of global optrA-carrying Enterococcus faecalis uncovers a common chromosomal hotspot for optrA acquisition within a diversity of core and accessory genomes. Microb. Genom. 2020, 6, e000350. [CrossRef] [PubMed]

32. Brenciani, A.; Morroni, G.; Vincenzi, C.; Manso, E.; Mingoia, M.; Giovanetti, E.; Varaldo, P.E. Detection in Italy of two clinical Enterococcus faecium isolates carrying both the oxazolidinone and phenicol resistance gene optrA and a silent multiresistance gene cfr. J. Antimicrob. Chemother 2016, 71, 1118-1129. [CrossRef] [PubMed]

33. Tian, Y.; Li, T.; Zhu, Y.; Wang, B.; Zou, X.; Li, M. Mechanisms of linezolid resistance in staphylococciand enterococci isolated from two teaching hospitals in Shanghai, China. BMC Microbiol. 2014, 14, 292. [CrossRef] [PubMed]

34. Hua, R.; Xia, Y.; Wu, W.; Yan, J.; Yang, M. Whole transcriptome analysis reveals potential novel mechanisms of low-level linezolid resistance in Enterococcus faecalis. Gene 2018, 647, 143-149. [CrossRef]

35. Morales, G.; Picazo, J.J.; Baos, E.; Candel, F.J.; Arribi, A.; Peláez, B.; Andrade, R.; de la Torre, M.A.; Fereres, J.; Sánchez-García, M. Resistance to Linezolid Is Mediated by the cfr Gene in the First Report of an Outbreak of Linezolid-Resistant Staphylococcus aureus. Clin. Inf. Dis. 2010, 50, 821-825. [CrossRef]

36. Belén, A.; Pavón, I.; Maiden, M.C.J. Multilocus Sequence Typing. Methods Mol. Biol. 2009, 551, 129-140. 